THE ASTROPHYSICAL JOURNAL, 566:512-520, 2002 February 10

(C) 2002. The American Astronomical Society. All rights reserved. Printed in U.S.A.

\title{
PROTON ACCELERATION IN ANALYTIC RECONNECTING CURRENT SHEETS
}

\author{
J. Heerikhuisen, ${ }^{1}$ Yuri E. Litvinenko, ${ }^{2}$ AND I. J. D. Craig ${ }^{1}$ \\ Received 2001 April 30; accepted 2001 September 9
}

\begin{abstract}
Particle acceleration provides an important signature for the magnetic collapse that accompanies a solar flare. Most particle acceleration studies, however, invoke magnetic and electric field models that are analytically convenient rather than solutions of the governing magnetohydrodynamic equations. In this paper a self-consistent magnetic reconnection solution is employed to investigate proton orbits, energy gains, and acceleration timescales for proton acceleration in solar flares. The magnetic field configuration is derived from the analytic reconnection solution of Craig and Henton. For the physically realistic case in which magnetic pressure of the current sheet is limited at small resistivities, the model contains a single free parameter that specifies the shear of the velocity field. It is shown that in the absence of losses, the field produces particle acceleration spectra characteristic of magnetic $X$-points. Specifically, the energy distribution approximates a power law $\sim \mathscr{E}^{-3 / 2}$ nonrelativistically, but steepens slightly at the higher energies. Using realistic values of the "effective" resistivity, we obtain energies and acceleration times that fall within the range of observational data for proton acceleration in the solar corona.
\end{abstract}

Subject headings: acceleration of particles - MHD - Sun: flares - Sun: magnetic fields Sun: particle emission

\section{INTRODUCTION}

Although soft X-ray emission resulting from strong plasma heating $\left(>10^{7} \mathrm{~K}\right)$ is probably the prime manifestation of the solar flare, it has often been suggested that flare energy may initially reside in nonthermal particles, typically in the form of electron and proton spectra extending to high energies. Protons, in particular, can be accelerated to several $\mathrm{GeV}$. The energization rate for protons above 1 $\mathrm{MeV}$ can reach $10^{34} \mathrm{~s}^{-1}$, and their energy content can exceed $10^{30}$ ergs (see Miller et al. 1997 for a review). Longduration solar gamma-ray flares are of particular interest in this regard, since they indicate the presence of continuously accelerated ions for several hours after the impulsive phase (e.g., Ryan 2000).

As yet there is no universal theory that accounts for the thermal and nonthermal signatures of the flare. There is, however, a general consensus that flare energy is derived from the rapid dissipation of strong-current sheets in the low solar corona. These sheets are intimately associated with magnetic reconnection, a resistive process thought to be the primary mechanism of magnetic energy release. Since a direct electric field is induced by the reconnecting magnetic field in the sheet, it is natural to ask whether the induced electric field can accelerate charged particles to typical nonthermal energies.

A large body of research has been devoted to the question of charged-particle orbits in reconnecting current sheets in the context of particle acceleration on the Sun and in the geotail (e.g., Speiser 1965; Martens 1988; Zhu \& Parks 1993; Litvinenko \& Somov 1993; Litvinenko 1996, 1997). Alternatively, particle orbits in the vicinity of a magnetic $X$-point have been analyzed and applied to the proton energization problem in flares (Bulanov \& Sasorov 1976; Bulanov 1980; Bruthwiler \& Zweibel 1992). Recent numerical simulations have also concentrated on the properties of

\footnotetext{
${ }^{1}$ Department of Mathematics, University of Waikato, Private Bag 3105, Hamilton, New Zealand.

${ }_{2}^{2}$ Institute for the Study of Earth, Oceans, and Space, University of New Hampshire, Durham, NH 03824-3525.
}

particle orbits in $X$-point fields (Mori, Sakai, \& Zhao 1998). The magnetic field in $X$-point current sheets plays a key role in particle acceleration: although the field cannot change the particle energy directly, it can limit the energy gain by changing the orbit and restricting the displacement along the electric field.

The magnetic configurations considered so far, however, suffer a major drawback. Although capturing the basic reconnection topology, they do not represent a quasi-steady reconnection solution. Some authors have tried to remedy this defect by tracing charged particles in magnetic field structures obtained by solving the magnetohydrodynamic (MHD) equations numerically (Schopper, Birk, \& Lesch 1999). Such strategies are invariably compromised by numerical resolution; in fact, particle gyroradii can be significantly smaller than the computational mesh. Numerical MHD experiments are also limited by the unnaturally large resistivities required to resolve the steep field gradients that accompany rapid magnetic merging.

Motivated by these difficulties, our aim is to investigate particle acceleration using an exact analytic magnetic reconnection geometry. Solutions are now available that describe steady-state, incompressible magnetic merging at arbitrary plasma resistivities, in both two and three dimensions (Craig \& Henton 1995; Craig et al. 1995). Numerical simulations (Heerikhuisen, Craig, \& Watson 2000) confirm that the steady-state solution agrees remarkably well with the properties of time-dependent reconnective current sheets. In the case of two-dimensional planar flows, there are two magnetic field components associated with the current sheet: only one of these - the planar flux pileup field - is capable of providing fast reconnection; the other component, aligned perpendicular to the flow, is dissipated at a slow Sweet-Parker rate by magnetic annihilation. It is the goal of this paper to investigate test particle orbits in the current sheet associated with the Craig \& Henton (1995) reconnection solution and apply the results to proton acceleration in solar flares.

Of related interest is the work of Kobak \& Ostrowski (2000), who recently employed the Craig \& Henton (1995) 
solution as a basis for a particle acceleration numerical experiment. Unfortunately, their work concentrates only on the slowly dissipating field components and neglects the physically more interesting "flux pileup" field. More critically, the solution they adopt (Craig et al. 1995) is valid for two-dimensional flows, while their choice of parameters implies a three-dimensional velocity field. Although Kobak \& Ostrowski's field configuration can no longer represent a solution of the MHD equations, it seems likely that their findings regarding turbulent particle acceleration are not seriously compromised.

A discussion of the analytic reconnection solution we employ is given in $\S 2$. Our main findings regarding proton acceleration are described in $\S 3$. We first analyze the acceleration properties of generic $X$-point magnetic structures before investigating the exact reconnection solution. We investigate both the form of the spectrum and the strength of the acceleration as a function of the effective plasma resistivity $\eta$. Application of the results to the high-energy acceleration of protons in the solar corona is presented in $\S 4$.

\section{THE RECONNECTION SOLUTIONS}

\subsection{Basic Equations}

We assume that the background plasma dynamics are governed by the steady-state, incompressible MHD equations for the magnetic field $\boldsymbol{B}$ and the velocity field $\boldsymbol{u}$. Using cgs units and normalizing the mass density to unity, the momentum equation can be written in the curled form

$$
(\boldsymbol{u} \cdot \nabla) \boldsymbol{\Omega}-(\boldsymbol{\Omega} \cdot \nabla) \boldsymbol{u}=\frac{1}{c}[(\boldsymbol{B} \cdot \nabla) \boldsymbol{J}-(\boldsymbol{J} \cdot \nabla) \boldsymbol{B}] .
$$

Here $\boldsymbol{\Omega}=\boldsymbol{\nabla} \times \boldsymbol{u}$ is the vorticity, and the current density $\boldsymbol{J}$ is obtained from Ampere's law $4 \pi J=c \nabla \times B$, with $\nabla \cdot B=0$.

The induction equation is given by

$$
\boldsymbol{\nabla} \times(\boldsymbol{u} \times \boldsymbol{B})+\bar{\eta} \nabla^{2} \boldsymbol{B}=0 .
$$

Here the magnetic resistivity is defined as

$$
\bar{\eta}=\frac{c^{2}}{4 \pi \sigma},
$$

and the electric conductivity is given, for instance, by the classical collision-based expression $\sigma \simeq 10^{7} T^{3 / 2} \simeq 10^{16}$ (cgs) for a fully ionized coronal plasma at temperature $T=10^{6} \mathrm{~K}$.

A self-consistent reconnection solution for the $\boldsymbol{u}$ and $\boldsymbol{B}$ fields is given in $\S 2.2$ below. The main focus of this paper is to investigate test-particle trajectories using the equation of motion

$$
\dot{\boldsymbol{p}}=q\left(\boldsymbol{E}+\frac{1}{c} \boldsymbol{v} \times \boldsymbol{B}\right), \quad \boldsymbol{p}=\gamma m \boldsymbol{v},
$$

where $v, q$, and $m$ are the velocity, charge, and rest mass of the particle, and $\gamma$ is the relativistic Lorentz factor. Given the $\boldsymbol{u}$ and $\boldsymbol{B}$ fields, the electric field responsible for the particle acceleration is easily calculated:

$$
\boldsymbol{E}=\frac{\bar{\eta}}{c} \nabla \times \boldsymbol{B}-\frac{1}{c} \boldsymbol{u} \times \boldsymbol{B} .
$$

\subsection{Magnetic Field Configuration}

It is convenient to measure physical variables in units appropriate to a typical coronal plasma. Nondimensionalization is done with respect to the following characteristic values:

$$
\begin{aligned}
& n_{c}=10^{9} \mathrm{~cm}^{-3}, \quad B_{c}=100 \mathrm{G}, \\
& L_{c}=10^{9.5} \mathrm{~cm}, \quad v_{\mathrm{A}}=10^{9} \mathrm{~cm} \mathrm{~s}^{-1},
\end{aligned}
$$

where $n_{c}$ is the coronal number density and $v_{\mathrm{A}}$ is the Alfvén speed. In these units $c=30$, and we have a nondimensional charge-to-mass ratio for protons of $9.067 \times 10^{7}$. It is important to note that the collision-based nondimensional resistivity is given by an extremely small inverse Lundquist number,

$$
\eta=\frac{\bar{\eta}}{L_{c} v_{\mathrm{A}}} \simeq 10^{-14} .
$$

As will be discussed later, however, due to the length scales and large current densities involved, the "effective" resistivity is expected to be enhanced by several orders of magnitude.

Let us now summarize the properties of the reconnection solution discovered by Craig \& Henton (1995) (see also Craig et al. 1995; Craig \& Fabling 1996). The magnetic and velocity fields can be written in terms of a superposition of a global background field $\boldsymbol{P}$ and a disturbance field $\boldsymbol{Q}$ in the following manner:

$$
\begin{aligned}
& \boldsymbol{u}=\boldsymbol{P}+\frac{\beta}{\alpha} \boldsymbol{Q}, \\
& \boldsymbol{B}=\frac{\beta}{\alpha} \boldsymbol{P}+\boldsymbol{Q} .
\end{aligned}
$$

This construction clearly exploits the inherent symmetry in the magnetic and flow fields. For the simplest case of a planar potential background and a one-dimensional disturbance field, the MHD equations are exactly satisfied by

$$
\begin{aligned}
& \boldsymbol{P}=\alpha[x,-y, 0], \\
& \boldsymbol{Q}=\left[\frac{Q_{0}}{\eta \mu} \operatorname{Daw}(\mu y), 0, \frac{\sqrt{\pi}}{2 \mu} Z^{\prime}(0) \operatorname{erf}(\mu y)+Z(0)\right],
\end{aligned}
$$

where

$$
\mu^{2}=\frac{\alpha^{2}-\beta^{2}}{2 \alpha \eta}, \quad \operatorname{Daw}(x)=\int_{0}^{x} \exp \left(t^{2}-x^{2}\right) d t
$$

is the Dawson function. Of the two internal scalar constants, $\alpha$ determines the strength of the background flow while $0<|\beta|<\alpha$ acts as a shear parameter that controls the angle of the $X$-point merging. Only one of the separatrices has magnetic field advected across it: the other lies along a coordinate axis (the $x$-axis), which also includes the center of the current sheet $y=0$ (see Fig. 2). It is the Dawson field component that is associated with reconnection: fields aligned normal to the flow dissipate slowly at the Sweet-Parker rate. Note that although dynamic numerical simulations (Heerikhuisen et al. 2000) have confirmed the overall veracity of the steady-state reconnection solution, certain refinements are required (namely, equalization and saturation as introduced below) to obtain a physical reconnection model. 


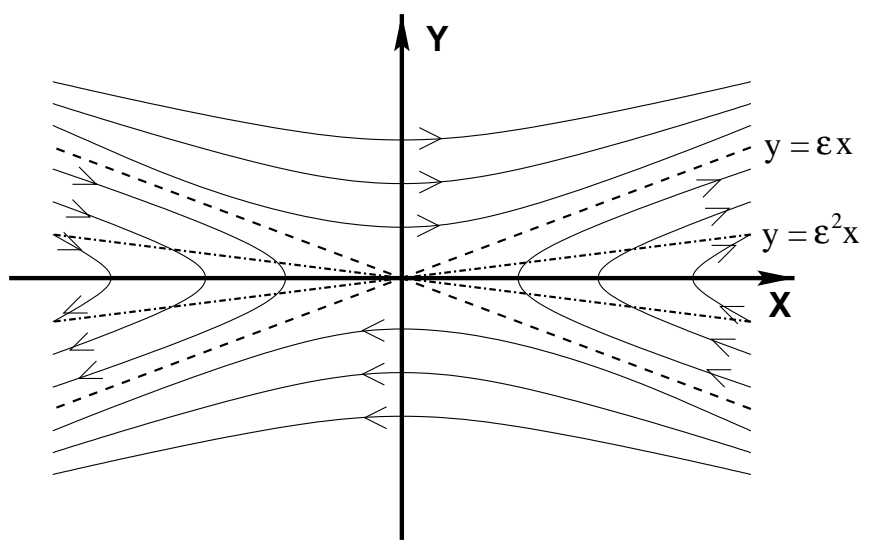

FIG. 1.-Schematic diagram of a typical $x$-point configuration (top) of the form $\boldsymbol{B}=B_{0}\left(y, \boldsymbol{\epsilon}^{2} x\right)$. The separatrices (dashed lines) are given by the lines $y= \pm \epsilon x$, while dash-dotted lines mark the region where $B_{y}$ exceeds $B_{x}$ and significant acceleration is possible. Current density is uniformly distributed over the entire domain.

Figures 1 and 2 provide a qualitative comparison of a classical $X$-point field with the sheared $X$-point solution discussed above. Given that the configurations have a superficially similar magnetic structure, we might anticipate similar particle-energy spectra to arise from both models.

\subsection{Application of the Reconnection Model}

Since we are concerned with fast magnetic reconnection, we begin by turning the nonplanar field component off, so that $Z(0)=Z^{\prime}(0)=0$. Reconnection now occurs at a neutral point (as opposed to a planar null) and the electric field is always aligned normal to the plane, that is,

$$
\boldsymbol{E}=-\frac{Q_{0}}{c} \hat{z} .
$$

The magnitude of the disturbance field, governed by $Q_{0}$, must be chosen to reflect the physical properties of the current sheet. One of the defects of the analytic reconnection model is that the magnitude of the disturbance field $Q$ is decoupled from the amplitude of the plasma flow defined by $\alpha$. A related difficulty is the apparently unbounded flux pileup in the limit $\eta \rightarrow 0$. These defects can be remedied by assuming physically reasonable equalization and saturation conditions.

The equalization assumption determines the flow amplitude: $\alpha$ is chosen so that the exhaust speed of the material

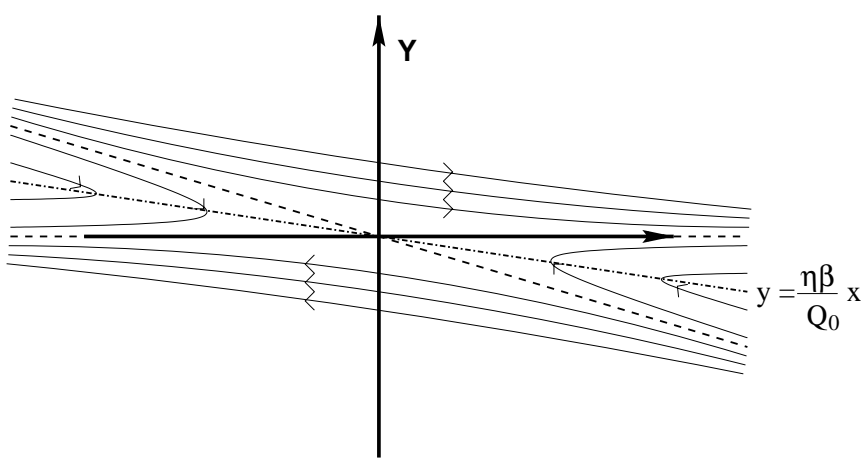

FIG. 2.-Schematic diagram of the Craig \& Henton solution described in section showing the field line (thin solid line) and separatrix (dashed line) in the $x y$-plane. The dash-dotted line represents the line along which $B_{x}$ vanishes and maximum acceleration occurs. Current is confined to a sheet aligned to the $x$-axis. expelled from the current sheet is determined by the local Alfvén speed based on the strength of the flux pile-up field. To prevent unbounded reconnection rates in the limit $\eta \rightarrow 0$ we also saturate the field at some physically appropriate level. In practice, this is achieved by choosing $Q_{0}$ so that the disturbance field in the sheet reaches a peak value corresponding to a dimensional value, say, of $B_{\max }=300 \mathrm{G}$. The equalization condition is then imposed (Litvinenko \& Craig 1999, 2000; Craig \& Watson 2000) by setting $\alpha=$ $B_{\max } / B_{c}=3$. We must also choose a suitable value for $\beta$ (remembering the restriction $0<|\beta|<\alpha$ ), and for all our work we take $\beta=2$. Since the value of $\alpha$ is set by the maximum of the Dawson function, we find that $Q_{0} \simeq 5 \sqrt{\eta}$.

The saturation and equalization assumptions have the physically desirable effect of removing much of the arbitrary parameterization from the reconnection model. Only the degree of shear, parameterized by $\beta$, remains free. Analytic arguments (Craig \& Watson 1999) now show that the peak magnetic field, electric field, and current sheet width scale with resistivity, independently of $\beta$, in the following way:

$$
B_{\max } \sim \eta^{0}, \quad E \sim \eta^{1 / 2} B_{\max }^{3 / 2}, \quad l \sim \eta^{1 / 2} B_{\max }^{-1 / 2} .
$$

Although the formal scalings with $\eta$ are the same as for Sweet-Parker, the amplification factor due to flux pile-up, say, $B_{\max } \sim 10$, gives strongly enhanced dissipation for even collisional resistivities.

More critically, the smallness of the collisional resistivity $\left(\eta \simeq 10^{-14}\right)$ implies the sheet becomes so thin that the MHD approximation breaks down, leading to the development of an enhanced anomalous resistivity. In such cases it is useful to invoke an "effective" resistivity several orders of magnitude larger than the classical value (see Litvinenko \& Craig 2000 and references therein). Although the effective resistivity is almost certainly influenced by local factors such as temperature and density, we consider plausible values mainly in the range $10^{-8} \leq \eta_{\text {eff }} \leq 10^{-6}$. In practice, resistive dissipation in the sheet could be enhanced by a variety of factors, for instance by the development of a current-driven instability.

The adoption of an effective resistivity clearly provides the simplest approach to modeling the super-Dreicer reconnecting field. More refined treatments, for example, including collisionless terms using a generalized Ohm's law (Bhattacharjee, Ma, \& Wang 1999), do not allow such a convenient analytic description. It seems likely, for instance, that the simple scaling arguments (see $\S 3$ ) that govern the overall properties of the accelerated particles would be compromised by more detailed treatments.

\subsection{Calculation of the Particle Spectra}

Let us assume that we have constructed the magnetic field configuration according to the prescriptions given above. This field is to remain static and undisturbed as far as particle acceleration is concerned, consistent with the short acceleration times of the particles relative to the Alfvénic reconnection timescale. Although the degree of shear determined by $\beta$ is arbitrary, we must remember that $\beta$ determines the angle of the $X$-point field, and that an appreciable perpendicular field component must be present in the current sheet for particle energies to remain finite. In practice we take $\beta=\frac{2}{3} \alpha$.

Although we assume a steady-state reconnection model, we could equally well have taken a time-dependent reconnection solution. In fact, the analytic model we assume is 
known to provide a good instantaneous picture of the reconnection region associated with global, time-dependent merging simulations (Heerikhuisen et al. 2000). It should be remembered that the analytic MHD solution we employ is characterized by the magnetic and velocity fields that increase with distance from the $X$-point and have infinite energy when integrated over all space. Hence, the model cannot be used to predict the total amount of energy released in the form of fast particles in the course of a flare. In this preliminary study, however, our main concern is to provide a simple and tractable platform for particle acceleration at realistic plasma resistivities, and not to provide a breakdown for the details of the magnetic energy release.

An embedded Runge-Kutta method is used to integrate the particle trajectories in time. The time step is determined by the local truncation error, which is related, in turn, to the local forces experienced by the particle. Each particle trajectory typically comprises millions of time steps. Although there are formally more accurate methods for solving orbit problems, it should be remembered that the chaotic nature of the trajectories (Chen \& Palmadesso 1986; Litvinenko 1993) negates the benefits of such schemes, particularly as we are interested in determining the associated energy spectrum.

\section{ACCELERATION SPECTRA}

\subsection{X-Point Field Models}

Recall that magnetic $X$-points are generally expected to produce well-defined particle acceleration spectra. Consider a field of the form

$$
\boldsymbol{B}=B_{0}\left(y, \boldsymbol{\epsilon}^{2} x, 0\right),
$$

as illustrated in Figure 1. The spectrum is deduced by noting that an initial burst of acceleration due to the electric field is effectively cut short by the local magnetic field. This occurs when the speed $v_{z}$ has built up sufficiently for the Lorentz force $\left(\sim v_{z} B_{\perp}\right)$ to gyrocapture the particle and thus limit the displacement along the $z$-axis and the energy gain.

Suppose a stationary particle is placed somewhere along the $x$-axis of the $X$-point field (eq. [3.1]). In this case the equation of motion (eq. [2.4]) reduces to

$$
\begin{aligned}
& \dot{p}_{x}=-\frac{q}{c} v_{z} B_{\perp}, \\
& \dot{p}_{z}=q E+\frac{q}{c} v_{x} B_{\perp},
\end{aligned}
$$

where $\boldsymbol{E}=E \hat{z}$, and $B_{\perp}=B_{\perp}(x)=B_{0} \epsilon^{2} x$ is the $y$-component of the field (eq. [3.1]). If particles are distributed on $y=0$ initially, all trajectories are limited to the $y=0$ plane.

It is easy to see that the particle displacement along the $z$-axis is limited. Assume for simplicity that the perpendicular magnetic field $B_{\perp}$ does not change significantly over the particle orbit. Since the peak kinetic energy occurs when $p_{z}=0$ and $p_{x}=-q B_{\perp} z / c=p$ is maximum, the displacement $z=z_{\max }$ must coincide with the instantaneous gyroradius of the particle. It follows that in the nonrelativistic case (Speiser 1965),

$$
\mathscr{E}=q E z_{\max }=2 m c^{2}\left(\frac{E}{B_{\perp}}\right)^{2} .
$$

This result may also be deduced by noting that the electric field is transformed away in the reference frame moving along the $x$-axis with the speed

$$
V=-\frac{c E}{B_{\perp}},
$$

a result that confirms that $2 \mathrm{~V}$ is indeed the maximum speed achieved by the particle in the rest frame. In the moving frame the particle simply gyrates at the fixed energy given by $\frac{1}{2} m V^{2}$.

An energy spectrum may be derived from the relation (Martens 1988)

$$
\frac{d n}{d \mathscr{E}}=\frac{d n}{d x} \frac{d x}{d \mathscr{E}},
$$

where $n$ is the particle number density. Since $B_{\perp} \sim x$, equation (3.4) gives the scaling

$$
\mathscr{E}=A x^{-2},
$$

where $A=2 m c^{2} E^{2} / \epsilon^{2}$. If we neglect relativistic effects and assume a uniform initial distribution $(d n / d x=K$, constant), then the energy spectrum should approximate

$$
\frac{d n}{d \mathscr{E}}=K A^{1 / 2} \mathscr{E}^{-3 / 2},
$$

which implies a characteristic power-law spectrum in the absence of any loss processes that might result in a steeper spectrum.

\section{2. $X$-Point Relativistic Particle Spectra}

It is interesting that the argument for a power law spectrum breaks down in the highly relativistic case. The kinetic energy, now manifested mainly as increase in mass rather than speed, can again be derived by transforming away the electric field. Using the relativistic form for the kinetic energy, namely $\mathscr{E}=\left(c^{2} p^{2}+m^{2} c^{4}\right)^{1 / 2}-m c^{2}$, modifies equation (3.4) according to

$$
\mathscr{E}=2 m c^{2} \frac{E^{2}}{\left(B_{\perp}^{2}-E^{2}\right)}
$$

for the case of a fixed perpendicular magnetic field and initially slow particles (Alekseyev \& Kropotkin 1970). Of course, the magnetic field experienced by the particle is not fixed over the capture phase. The effect of the inhomogeneous magnetic field is particularly noticeable for protons released close to the neutral point, where even slight deflections in the $x$-direction lead to the particle experiencing strong relative enhancements in $B_{\perp}$ (Bulanov \& Sasorov 1976). In spite of this limitation, equation (3.9) should remain qualitatively valid, indicating that the particles can gain very large energies in the vicinity of the $X$-point defined by the condition $B_{\perp}(x)<E$. Of interest is the fact that relativistic effects, which give rise to longer acceleration times for particles near the neutral point, will tend to negate the inhomogeneous $B_{\perp}$, which shortens the acceleration time. Hence, the evidence suggests that the nonrelativistic constant field approximation (eq. [3.4]) can provide a good predictor for relativistic inhomogeneous magnetic field acceleration (see Fig. 3 and discussion below).

It is a simple matter to verify these observations numerically for the reference $X$-point field (eq. [3.1]). In order to provide a comparison, we set the $X$-point angle specified by $\epsilon$ to be the same as the corresponding separatrix angle in the exact reconnection solution (see Heerikhuisen et al. 2000). With this specification, $B_{\perp}$ is not quite strong enough 


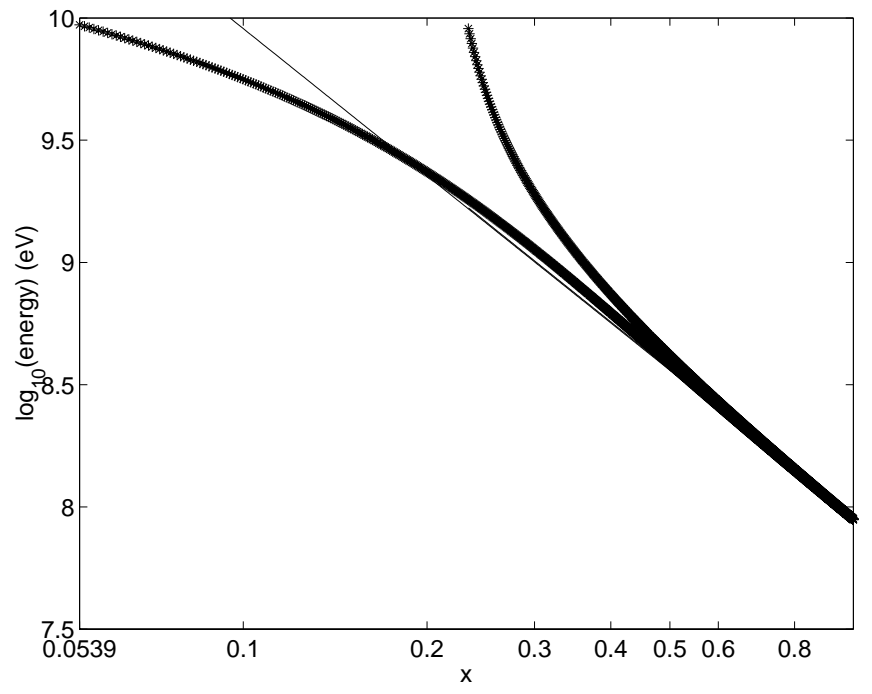

FIG. 3.-This figure shows the relationship between initial position and maximum energy gain for particles released along the $x$-axis of the $X$-point configuration (eq. [3.1]). The upper solid line represents the simpler case where $B_{\perp}$ remains fixed over a particle's entire orbit, while for the lower solid $B_{\perp}$ this simplification is not made. The thin straight line represents the nonrelativistic scaling predicted by eq. (3.7).

to capture the majority of particles and so, to obtain suitable test conditions, we adjust the level of magnetic field (by a factor of 10) independently of the electric field.

Figure 3 shows the computed relationship between initial position and maximum energy. The central straight line gives the scaling predicted by the nonrelativistic approximation (eq. [3.4]). This scaling is accurate until relativistic effects kick in at energies above $10^{8.5} \mathrm{eV}$, which corresponds to $v / c \simeq \frac{2}{3}$. The solid line above this represents a set of particle orbits for which, to maintain strict fidelity with the analytic scaling argument, $B_{\perp}$ is fixed to its initial value on each trajectory. This curve precisely coincides with the relativistic prediction (eq. [3.9]). The lower solid line in Figure 3 represents the real $X$-point solution, in which $B_{\perp}$ increases according to the $x$-position of the particle. This effect clearly decreases the expected energy gain of relativistic particles started close to the neutral point where the initial $B_{\perp}$ is weak: prior to capture these particles experience everincreasing $B_{\perp}$ fields because of their motion in the $x$ direction.

Figure 4 shows the spectrum obtained from the lower plot of Figure 3. The reference line represents the prediction in equation (3.8), and it is clear that the theoretical powerlaw scaling is quite well reproduced. A slight steepening is evident at higher energies corresponding to a shallowing of $\mathscr{E}(x)$ (see Fig. 3). The high-energy endpoint that lies well above the reference line corresponds to the maximum energy acquired by all noncaptured particles.

\subsection{Results for the Reconnecting Current Sheet}

Since the exact reconnection solution due to Craig \& Henton (1995) provides a model for $X$-point reconnection, we might expect to achieve a spectrum in accordance with prediction (3.8). There are, however, two key assumptions in the analytic argument that could compromise the energy scaling. In the first place, the calculated distribution is based on particle capture: if the Lorentz force is too weak to halt the acceleration before the particle exits the region, then such a particle will acquire the maximum energy $\mathscr{E}_{\max }=$

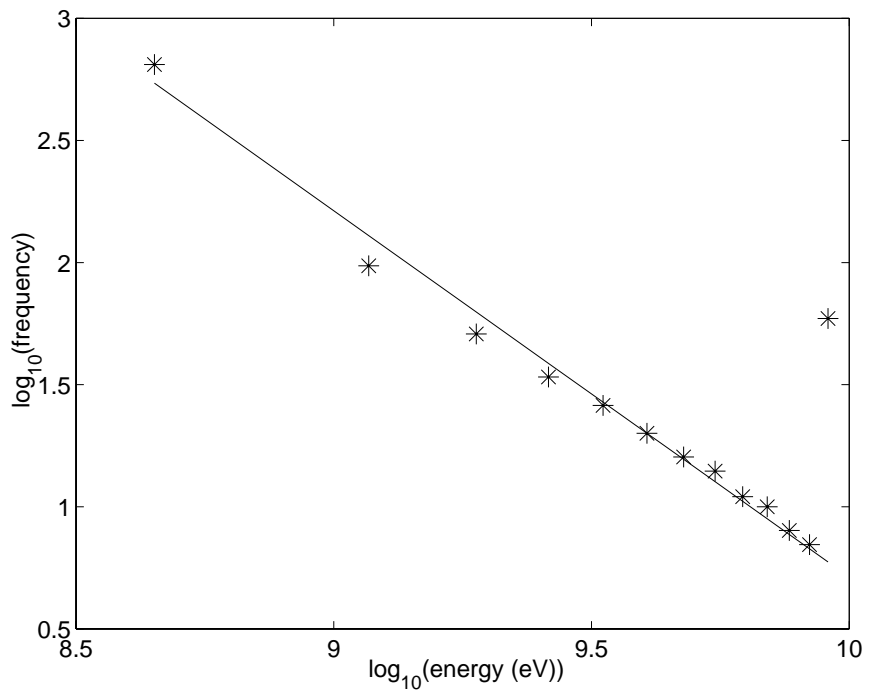

FIG. 4.- Spectrum obtained from the lower line of Fig. 3, corresponding to the maximum energy attained by distributing 1000 particles uniformly along the $x$-axis and releasing them from rest in the $X$-point field (eq. [3.1]).

$q E L_{c}$ defined by the size $L_{c}$ of the computational box (see eq. [3.4]). This situation will always apply for some interval close to the neutral point. In fact, situations could arise where this interval covers the entire region and no spectrum would be achieved.

The analytic argument also assumes that initial conditions are chosen so that there is immediate downward acceleration in the weak-field region where $B_{\perp}>B_{x}$. In fact, this assumption, although unrealistic, may not be too critical, since any particle placed near the current sheet will migrate into the weak-field region due to the electric drift associated with the dominant reconnecting magnetic field $B_{x}$ outside the current sheet (see Fig. 2) and the constant electric field in the $-\hat{z}$-direction. In this case a slow inward-drift phase should precede the rapid $\boldsymbol{E}$-field acceleration within the sheet.

Figure 5 shows the energy versus $x$-position diagram for the exact MHD solution. Three values of the resistivity are plotted. There is close agreement with the analytic nonrelativistic scaling at low energies-here the individual curves are almost indistinguishable - but significant departures at relativistic energies are present. Note, however, that each curve represents a close approximation to the $X$-point model in the lower plot of Figure 3. The upper cutoff in each $\mathscr{E}(x)$ plot reflects the maximum energy gain (eq. [3.4]) of a noncaptured particle, and since $E \sim \eta^{1 / 2}$, the maximum energy gain must also scale in this way.

To interpret these results, first note that the low-energy scaling is consistent with the capture condition, being invariant with $\eta$. That is, in the saturated solution, both $E$ and $B_{\perp}=B_{y}$ (at fixed $x$ ) scale as $\eta^{1 / 2}$. Thus, for a given initial position, the energy should remain invariant with $\eta$, at least provided that $x$ is large enough for $B_{\perp}$ to be effectively constant prior to capture. At high energies, the scalings must separate to reflect the increased direct field acceleration of noncaptured particles. In turn, each energy curve has to be consistent with the form of Figure 3.

Figure 6 shows the spectrum computed from the exact Craig-Henton solution. The spectrum, comprising 1000 particles released with some small random thermal energy 




FIG. 5.-Final energy with initial position for 50 particles evenly distributed along the positive $x$-axis of the Craig-Henton field of $\S 2.2$ and started from rest. The three lines represent, from top to bottom, $\eta=10^{-6}$, $\eta=10^{-7}$, and $\eta=10^{-8}$, respectively. The solid line gives the scaling prediction (eq. [3.7]). Note that we have only plotted energy of the particles that are captured within our $-L_{c}$ to $L_{c}$ cube.

of $0.1 v_{\mathrm{A}}$, is strikingly similar to the $X$-point spectrum of Figure 4. Again there is slight steepening due to the shallowing of $\mathscr{E}(x)$ (see Fig. 5) and a spike at the upper energy cutoff corresponding to noncaptured particles.

The fact that the heuristic $X$-point spectrum manages to reproduce the main features of the steady-state reconnection solution confirms the assumption of previous studies: that $X$-point current sheet models can provide a useful platform for particle acceleration investigations. It should be remembered, however, that the advantage of the exact solution used here is that the analysis is unambiguous, in the sense that it is uncompromised by extraneous parameterizations.

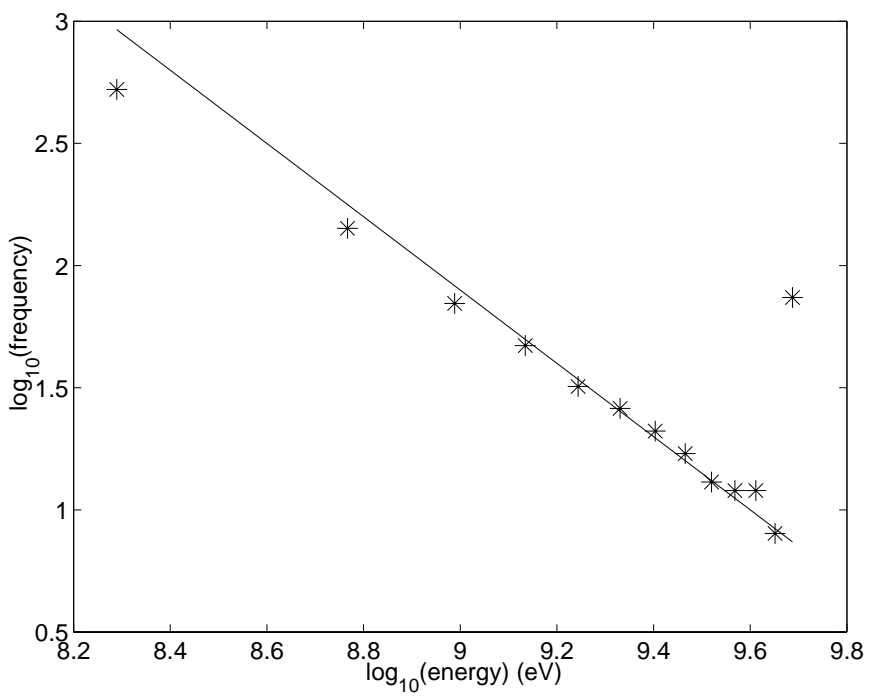

FIG. 6.- Spectrum corresponding to the final energy of 1000 particles distributed uniformly along the center of the current sheet of the CraigHenton field. Here we have used $\eta=10^{-7}, \alpha=3$, and $\beta=2$, and each particle was given an initial speed $0.1 v_{\mathrm{A}}$ in a random direction.

\subsection{Resistive Scalings}

How does the maximum kinetic energy scale with resistivity? Note that the nondimensional electric field always saturates according to $E=\eta^{1 / 2} B_{\max }^{3 / 2}$ (Craig \& Watson 2000). Thus, using $\mathscr{E}_{\max }=q E z_{\max }$ with $z_{\max }=L_{c}$ yields the limit

$$
\mathscr{E}_{\max } \simeq 3 \times 10^{12} \eta^{1 / 2} B_{\text {max }}^{3 / 2} \mathrm{eV} .
$$

This suggests that inverse Lundquist numbers as small as $10^{-10}$ could be sufficient (taking $B_{\max }=10$ corresponding to localized sheet fields of $10^{3} \mathrm{G}$ ) to produce $\mathrm{GeV}$ protons. Such fields are in fact capable of producing flare-like Ohmic decay rates of $10^{28} \mathrm{ergs} \mathrm{s}^{-1}$ (Litvinenko \& Craig 2000).

Turning now to the acceleration time of a particle captured in the sheet, we recall that the acceleration length is simply the gyroradius $c p / q B_{\perp}$. For nonrelativistic motion, the acceleration time is one-half the gyroperiod:

$$
\tau_{\mathrm{acc}}=\frac{\pi m c}{q B_{\perp}} \sim \eta^{-1 / 2} .
$$

Note that although the gamma factor must be included in the case of relativistic motion,

$$
\tau_{\mathrm{acc}}=\frac{\pi m c}{q B_{\perp}} \frac{B_{\perp}^{2}+E^{2}}{B_{\perp}^{2}-E^{2}},
$$

the (formal) scaling with resistivity due to $B_{\perp}(x)$ is not undone. The relativistic factor does, however, alter the scaling coefficient. This result is confirmed by the acceleration times plotted in Figure 7 for the Craig-Henton model. The weaker $\boldsymbol{E}$-field acceleration at low resistivities leads to longer acceleration times.

Of course, particles initially along the center line of the sheet ( $x$-axis) must first drift into the weak-field region before being accelerated. An estimate of this drift time can

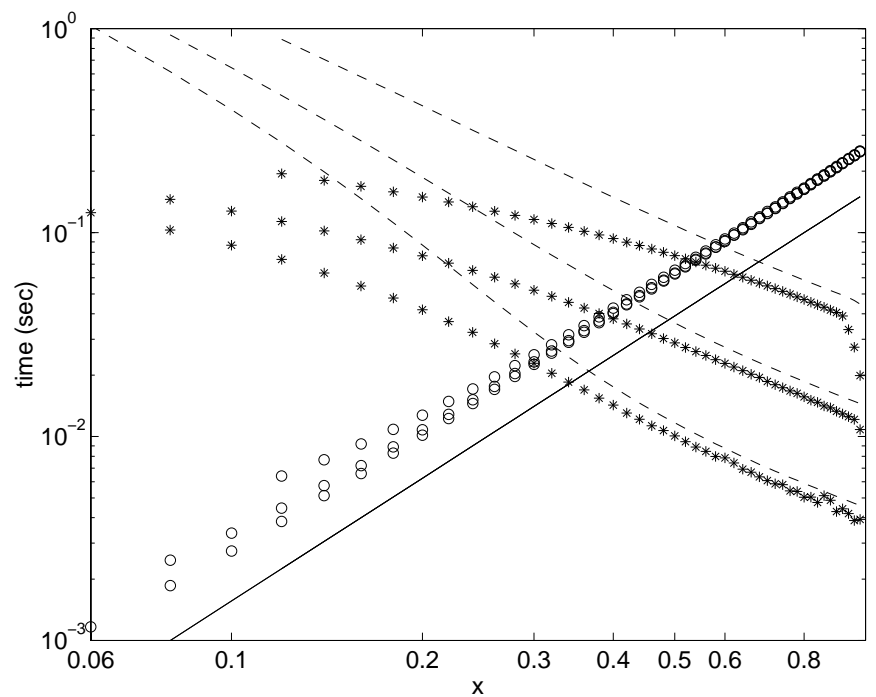

FIG. 7.-Drift time (open circles) and acceleration time (asterisks) for three sets of particle simulations for $\eta=10^{-6}, 10^{-7}$, and $10^{-8}$. The drift time is the same for all three runs (some slight error at small values of $x$ is caused by the error in estimating when the drift phase has finished), and is approximated by eq. (3.13), which is plotted as the solid line. The dashed lines represent the analytic prediction, eq. (3.12). 
be made by taking the drift distance $\beta \eta x / Q_{0}$ (see Fig. 2) and dividing by the initial drift speed $v_{\text {drift }} \simeq Q_{0} /(\beta x)$ to give

$$
\tau_{\mathrm{drift}} \simeq \frac{\eta \beta^{2}}{Q_{0}^{2}} x^{2} .
$$

The predictions (3.12) and (3.13) are plotted along with the computed values for $\eta=10^{-6}, 10^{-7}$, and $10^{-8}$ in Figure 7 . The acceleration time prediction (3.12) agrees well for particles started away from the neutral point, since for these the implicit assumption of constant $B_{\perp}$ is approximately valid over the acceleration phase of each particle. The drift time prediction (3.13) is much cruder, but is clearly still a consistent measure over the entire sheet.

The flux spectrum is of interest observationally. The flux of protons into the acceleration region will be given by

$$
\mathscr{F}_{\text {in }}=2 L_{z} \int_{x_{\min }}^{x} v_{\mathrm{in}} n_{\mathrm{in}} d x
$$

and will be the same as the outward flux of accelerated particles, assuming that all particles enter the acceleration process. Using $v_{\text {in }}=v_{\text {drift }} \simeq Q_{0} /(\beta x), \mathscr{E}_{\text {in }}=m v_{\text {in }}^{2} / 2$, and a uniform particle density $n_{\text {in }}$, the flux may be written as

$$
\mathscr{F}\left(\mathscr{E}_{\text {in }}\right)=\frac{L_{z} n_{\text {in }} Q_{0}}{\beta} \int_{\mathscr{E}_{\text {in }}}^{\mathscr{E}_{\max }} \mathscr{E}^{-1} d \mathscr{E} .
$$

However, we are interested in the outward flux in terms of the final energy of the particles. We know from equation (3.4) and the expression for $v_{\text {in }}$ used above that the inward and outward energies are related in the following way:

$$
\mathscr{E}_{\text {out }}=\frac{4 Q_{0}^{2}}{\beta^{2} \eta^{2}} \mathscr{E}_{\text {in }}
$$

Hence, the particle flux in terms of the final energy will be

$$
\mathscr{F}(\mathscr{E})=\frac{L_{z} n_{\text {in }} Q_{0}}{\beta} \ln \left(\frac{\mathscr{E}_{\text {max }}}{\mathscr{E}}\right),
$$

where $\mathscr{E}$ is the final kinetic energy of each particle. It is easiest to interpret this equation nondimensionally by taking $L_{z}=1, n_{\mathrm{in}}=1, Q_{0}=5 \sqrt{\eta}$, and $\beta=2$. Concrete numbers are then obtained by taking $\mathscr{E}_{\max }=q E z_{\text {max }} \simeq 2 \sqrt{\eta}$ $\times 10^{13} \mathrm{eV}$ and the reference flux $n_{c} v_{\mathrm{A}} L_{c}^{2}=10^{37} \mathrm{~s}^{-1}$ to give

$$
\mathscr{F}(\mathscr{E}) \simeq 2.5 \times 10^{37} \sqrt{\eta} \ln \left(\frac{1.6 \sqrt{\eta} \times 10^{13}}{\mathscr{E}}\right) \mathrm{s}^{-1},
$$

where $\mathscr{E}$ is also measured in eV. From this we can see that the expected flux for protons above $1 \mathrm{MeV}$ will be about $1.8 \times 10^{34}$ for $\eta=10^{-8}$, with about $7 \%$ of these being above $1 \mathrm{GeV}$.

It should be emphasized that the present particle acceleration model is self-consistent only for individual testparticle orbits, not necessarily for high-intensity particle beams. Formally, the above estimate for the proton flux should be multiplied by an efficiency factor, indicating the fraction of particles entering the acceleration process.

It has long been recognized that strong particle currents, as implied say by hard X-ray observations, pose severe theoretical difficulties (see Martens 1988). Compare, for example, the MHD reconnection current $\left(I_{R}\right)$, defined by Ampere's law for the flux pileup sheet, with the current associated with free-streaming (noncaptured) chargedparticle flux $\left(I_{P}\right)$. For $\eta=10^{-8}$, we find that for an efficiency factor of order unity, $I_{P}$ can exceed $I_{R} \simeq 10^{12.5} \mathrm{~A}$ by an order of magnitude. Since beam currents of this intensity must feed-back on the reconnection model, it is safest to regard flux magnitudes such as $I_{P}$ as preliminary upper estimates, rather than concrete theoretical predictions. Although it is beyond the scope of this paper to attempt such predictions, it appears likely that a more detailed treatment taking into account finite acceleration efficiency and return currents would lead to a physically plausible total electric current in the sheet $I_{P} \simeq I_{R}$.

\subsection{Orbit Properties}

The interpretations in the previous sections are reinforced by studying individual particle orbits. Figure 8 shows part of a typical particle orbit, comprising well-defined inward drift, acceleration in the reconnection region, and capture phases. The particle starts from rest at $(0.4,0,0)$ and initially drifts slowly in the $-\hat{\boldsymbol{y}}$-direction, gyrating around the relatively strong reconnecting field $B_{x}$. When the particle reaches the weak-field region where $B_{x} \approx B_{\perp}$, it is accelerated rapidly downward by the strong $\boldsymbol{E}$ field. This phase ends when the particle speed is sufficient for the Lorentz force to recapture the energized particle. In the final phase the particle exits the source volume, spiraling in the $y z$ plane, through the surface $x=1$.

It should be noted that in all steady-state planar reconnection solutions, the electric field is constant everywhere (see eq. [2.11]). This begs the question, "is it possible to acquire significant acceleration outside the current sheet?" We have argued that significant acceleration is only possible if test particles can gain entry, if not by in situ placement then by slow drift, into the weak-field exhaust of the reconnection mechanism.

More specifically, test particles placed well outside the current sheet will either drift into the exhaust region and get accelerated in much same way as particles placed inside the sheet or simply drift out of the domain without a significant energy gain. Figure 9 shows the projection of such orbits in the $x y$-plane (there is little motion in the $\hat{z}$-direction). As

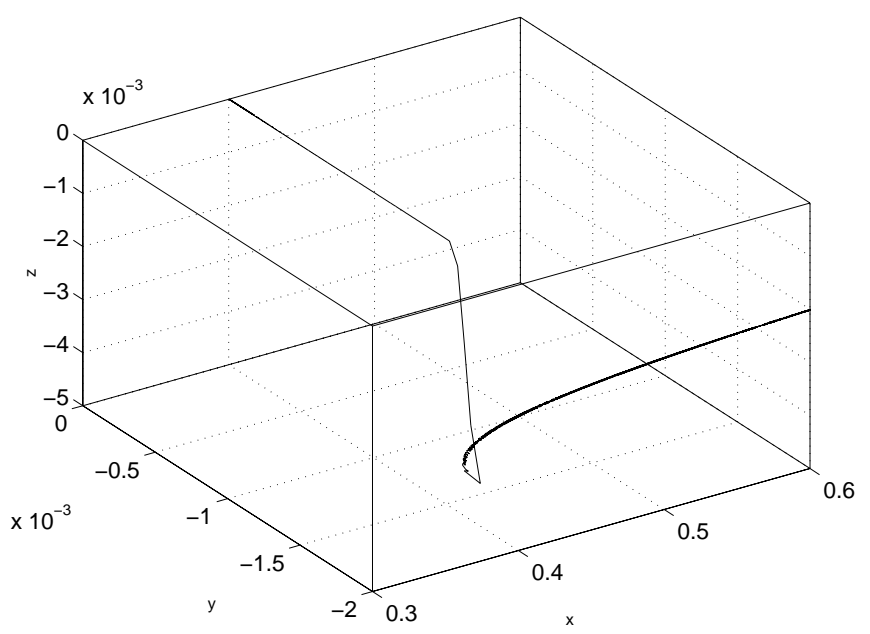

FIG. 8.-Orbit of particle started from rest at $(0.4,0,0)$. Note the initial drift phase (motion in $y$ ), followed by the acceleration phase (motion in $z$ ), and concluded by the exit phase (motion in $x$ ). 




FIG. 9.- Trajectories of nine particles started well outside the current layer (central jagged region). The dashed lines depict magnetic field lines. We find that only those that encounter the current sheet achieve significant acceleration.

expected, the particles drift in the $\boldsymbol{E} \times \boldsymbol{B}$ direction and attain little energy outside the current sheet where the magnetic field is comparatively large. Only the particles that drift into the current sheet (originating in the lower left and upper right quadrants) will achieve significant acceleration.

\section{APPLICATION TO SOLAR FLARES}

Observations of events in the solar corona have led to the conclusion that the bulk of accelerated protons have energies within the range $0.1-10 \mathrm{MeV}$. Earth-based detectors now and then record protons coming from the sun with energies in the $1-5 \mathrm{GeV}$ range (Ryan 2000). The particles with the highest energies typically come from the large gradual events that are most likely a consequence of magnetic field relaxation following a coronal mass ejection (CME). The CME is likely to generate a shock wave that contributes to particle acceleration. Significant observational evidence, however, suggests that at least some particles are energized in the current sheet formed in the wake of the CME (Klein \& Trottet 2001).

It has previously been shown (Litvinenko \& Craig 2000) that the Craig-Henton reconnection solution provides a physical model that is capable of meeting the bulk flare energy requirements, assuming a realistic turbulent resistivity that corresponds to the inverse Lundquist number of order $\eta \sim 10^{-8}$. The analysis of the present paper demonstrates that the same current sheet may be the source of energetic protons in large solar flares. In particular, energies of order $\eta^{1 / 2} 10^{13} \mathrm{eV}$ are possible using flux pileup fields of order $300 \mathrm{G}$ (Fig. 5). Another prediction of the model is that the direct electric field acceleration in the current sheet provides not only the required proton energy but also the rate of energy gain. Equation (3.12) indicates that a typical proton acceleration timescale is of order $10^{-2} \mathrm{~s}$, assuming the transverse magnetic field in the sheet of a few gauss or smaller. This compares favorably with timescales $0.01-0.1 \mathrm{~s}$ of rapid variation of flare gamma-radiation produced by energetic protons (see Litvinenko \& Somov 1995 and refer- ences therein). The observed variability is likely to reflect a regime of bursty magnetic reconnection.

Solar observations also indicate the existence of an energy spectrum with a power-law or Bessel-function shape (Miller et al. 1997). Traditional models, using relatively weak $X$-point fields, account for such a spectrum via the magnetic field component perpendicular to the current sheet. Such models do not, however, represent quasi-steady solutions to the MHD equations, nor do they possess the physical features - such as Alfvénic shear flows, magnetic sling shots, and rapid Ohmic dissipation - expected of a plausible reconnection mechanism. It is encouraging, therefore, that the present model generates a power-law spectrum that can be understood in terms of $X$-point acceleration, for which $d n / d \mathscr{E} \sim \mathscr{E}^{-3 / 2}$. Of course a steeper spectrum is possible if the processes of energy and particle losses at the reconnection site are taken into account.

In analyzing particle spectra, we have noted that predictions obtained by using a nonrelativistic approximation and assuming constant perpendicular magnetic field accord reasonably well with simulations in which we make neither assumption. From this it is clear that the relativistic effects and inhomogeneity in $B_{\perp}$ cancel each other out to some degree. We should also emphasize that although simple $X$-point models provide qualitatively similar results to the steady-state reconnection solution, these contain a number of arbitrary factors that cannot be interpreted in terms of the physical properties of reconnecting current sheets. For example, it is not clear how the field strength should be normalized or what $X$-point angle should be used. We have shown here that detailed quantitative predictions can be made, unhindered by extraneous parameterizations, using an exact reconnection model.

Our field model is essentially two dimensional. We can expect more general three-dimensional solutions to provide richer acceleration spectra. It is expected, in particular, that electrons can be easily magnetized by the magnetic field component $B_{z} \approx 0.1 B_{x}$ (Litvinenko 1996), resulting in efficient electron acceleration to energies corresponding to $\mathrm{X}$-ray and gamma emission. Hence, the reconnecting magnetic field geometry can be responsible for the electron-toproton ratio as a function of energy. Another possible extension of our approach is through relaxing the steadystate assumption, using the available time-dependent generalizations of the Craig-Henton model to investigate whether the accelerated particle spectra and composition can be a signature of time-dependent reconnection. The results already obtained, however, should contribute to a better understanding of magnetic energy conversion and particle acceleration in solar flares. Of particular interest as far as applications are concerned is the time-extended proton acceleration that sometimes occurs in the corona for hours in large gradual solar flares.

To conclude, we have given the first study of test particle orbits in realistic reconnecting current sheets, described by an exact MHD solution for the magnetic fields and plasma flows. As an application, we considered proton orbits in two-dimensional geometries and demonstrated that nonthermal protons observed in solar flares can indeed be generated in a large-scale current sheet.

This work was partly supported by NSF grant ATM9813933, NASA grant NAG 5-7792, and Marsden Fund Grant 96-UOW-MIS-0006. 


\section{REFERENCES}

Alekseyev, I. I., \& Kropotkin, A. P. 1970, Geomagn. Aeronomy, 10, 755

Bhattacharjee, A., Ma, Z. W., \& Wang, X. 1999, J. Geophys. Res., 104, 14543

Bruthwiler, D. L., \& Zweibel, E. G. 1992, J. Geophys. Res., 97A, 10825

Bulanov, S. V. 1980, Soviet Astron. Lett., 6, 206

Bulanov, S. V. \& Sasorov, P. V. 1976, Soviet Astron., 19, 464

Chen, J., \& Palmadesso, P. J. 1986, J. Geophys. Res., 91A, 1499

Craig, I. J. D., \& Fabling, R. B. 1996, ApJ, 462, 969

Craig, I. J. D., Fabling, R. B., Henton, S. M., \& Rickard, G. J. 1995, ApJ, 455, L197

Craig, I. J. D., \& Henton, S. M. 1995, ApJ, 450, 280

Craig, I. J. D., \& Watson, P. G. 1999, ApJ, 516, 924 2000, Sol. Phys., 194, 251

Heerikhuisen, J., Craig, I. J. D., \& Watson, P. G. 2000, Geophys. Astrophys. Fluid Dyn., 93, 115

Klein, K. L., \& Trottet, G. 2001, Space Sci. Rev., 95, 215

Kobak, T., \& Ostrowski, M. 2000, MNRAS, 317, 973

Litvinenko, Y. E. 1993, Sol. Phys., 147, 337 1996, ApJ, 462, 997

. 1997, Phys. Plasmas, 4, 3439

Litvinenko, Y. E., \& Craig. I. J. D. 1999, Sol. Phys., 189, 315 2000, ApJ, 544, 1101

Litvinenko, Y. E., \& Somov, B. V. 1993, Sol. Phys., 146, 127 1995, Sol. Phys., 158, 317

Martens, P. C. H. 1988, ApJ, 330, L131

Miller, J. A., et al. 1997, J. Geophys. Res., 102, 14631

Mori, K., Sakai, J., \& Zhao, J. 1998, ApJ, 494, 430

Ryan, J. M. 2000, Space Sci. Rev., 93, 581

Schopper, R., Birk, G. T., \& Lesch, H. 1999, Phys. Plasmas, 6, 4318

Speiser, T. W. 1965, J. Geophys. Res., 70, 4219

Zhu, Z., \& Parks, G. 1993, J. Geophys. Res., 98A, 7603 